gathered from participants, carers, health and social care staff provided a more nuanced understanding of the impact of a nonclinical intervention and led to the development of best practice guidelines for those working in museums and health. The research, which is funded by the Arts and Humanities Research Council, has led to further funding to investigate the value of museum engagement in social prescribing. 'Museums on Prescription' will connect socially isolated, vulnerable and lonely older adults, referred through local NHS and Local Authority Adult Social Care services, to partner museums in Central London and Kent. A combination of qualitative and quantitative methods will be used for analysis of interview data and measures of wellbeing and social isolation taken before, during and after 10 weekly, two-hour, museum sessions, and at three and six month telephone follow-ups, to provide insights into non-clinical prescriptions and a psycho-social model of health rather than reliance upon a purely medical model.

\section{OP THE USE OF MIXED METHODS IN ASSESSING WELLBEING BENEFITS DERIVED FROM A HERITAGE- IN-HEALTH INTERVENTION WITH HOSPITAL PATIENTS AND CARE HOME RESIDENTS}

HJ Chatterjee, ${ }^{1,2 *} \mathrm{~L}$ Thomson'2. 'Department of Genetics, Evolution and Environment, Division of Biosciences, School of Life and Medical Sciences, University College London, UK; ${ }^{2}$ UCL Public and Cultural Engagement, University College London, UK

*Presenting author.

10.1136/bmjopen-2015-UCLSymposiumAbstracts. 7

The role of non-clinical interventions in health and social care is attracting increased interest as the value of community based assets in public health gains support. For the last six years University College London researchers have explored the advantages of a mixed methods approach to assess the therapeutic benefits of museum-focused interventions in healthcare settings including hospital wards and care homes. Using a combination of qualitative grounded theory and thematic analysis together with quantitative measures has resulted in a robust appraisal of the potential of handling and discussing museum objects in terms of positive effects on cognitive stimulation, health and wellbeing. Measures consisting of the Positive and Negative Affect Schedule (PANAS) and Visual Analogue Scales (VAS) taken before and after object handling sessions of around 40 minutes showed increases in positive emotion and decreases in negative emotion with improvements in subjective wellbeing and happiness. Qualitative analysis of audio recordings from the sessions were used to account for how improvements in wellbeing were brought about and revealed that mutual exploration of the objects led to deeper thinking and meaning making, and acted as a distraction from the clinical environment. Interview data 